ROLE OF DELAY IN SAMPLE PROCESSING AND OTHER FACTORS IN INTERPRETATION OF CEREBROSPINAL FLUID (CSF) CELL

686 COUNTS IN TRAUMATIC LUMBAR PUNCTURE (LP) FOR SUSPECTED MENINGITIS (M). Bernhard L. Wiedermann, ChrisAnna
Morgan, Lisa A. Kammerman and Pamela R. Getson. (Spon
by Wm. J. Rodriguez) Children's Hospital Nat Med Ctr and George Washington University School of Medicine \& Health Sciences, washington, D.C.

276 LP attempts were examined prospectively by physician questionnaire and medical record review. Factors occurring at the time of LP as well as time required for CSF receipt at the laboratory (DEL) were compared with clinical and laboratory variables. Occurrence of traumatic LP increased with decreasing experience level of person performing LP $(p=0.04)$ and with younger patient age $(p=0.0002)$. DEL averaged $28 \mathrm{~min}$, with $73 \%$ of samples $\leq 30$ min DEL but $11.5 \%$ 2 60 min DEL. A standard correction formula using CSF RBC count and blood RBC and WBC counts for specimens with $210,000 \mathrm{CSF}$ RBC resulted in $11 / 15$ (73\%) of non-M samples with negative corrected CSF WBC. Percent fresh RBC ranged from 0 to 100 and did not correlate with $D E L(r=0.02)$. 2 of $48 \mathrm{M}$ patients had negative or normal corrected CSF WBC with this formula. DEL did not correlate with corrected CSF WBC. Linear regression analysis with multiple independent varfables (CSF RBC, blood WBC, blood RBC, CSF WBC, and DEL) failed to produce a meaningful correction formula ( $r=0.47$ with best $f i t)$. Over-correction of CSF WBC is likely to occur in traumatic LP and is not explained by DEL. Determination of percent fresh RBC in CSF is not helpful in interpretation of traumatic LP in suspected M. Mathematicl models to correct CSF WBC for trauma are not clinically useful and should not be used.

\section{A POPULATION - BASED STUDY OF CHILDREN EXPOSED TO} CHLORIDE DEFICIENT FORMULA Anne D. Willoughby, Barry

687 Storr, Nancy Feter M. Vietze, Anita M. Hocker, Carla Btorr, Nancy F. Gist, George G. Rhoad

In 1978 and 1979 Syntex, Inc. manufactured two soy-based infant formilas witich lacked adequate chloride. A number of small studies have suggested that children who ingested these formulas may have suffered Iong-term adverse effects secondary to this exposure. However, these studies involved relatively few subjects who were selected in a fashion subject to
relion substantial bias. This study ascertained the infant formulas used by the first and second graders attending public school in one county in the Southeastern United States. Children who were exposed to a defective infant formula were matched to two children who ingested chloride-sufficient soy-based formulas as infants. Matching variables included age, race, sex, socioeconomic status and birth weight. All children $(n=108)$ received a standarized test of intellectual ability, the McCarthy Scales, which were administered by an examiner biinded to the exposure status of each child. Significant adjusted mean differences between the score on the General Cognitive Index (diff. $=5.7$, one-tailed $\mathrm{p}=.05$ ) and the Quantitative Scale (diff. $=3.1$, one-tailed $p<.05$ ) were documented with the exposed children scoring lower than their matched controls. Decrements in the performance of exposed children were noted on the Perceptual-Performance (diff. $=3.2$. one-tailed $p<.15$ ) and Motor (diff. $=3.3$, one-tailed $p<.1$ ) Scales although these were not statistically significant. These data reinforce concern about the long-term outcome of children exposed to these formulas.

EVALUATION OF AN EFFICIENT INEXPENSIVE TEACHING MODEL FOR VENOUS CUT DOWN. Jerry J. Zimmerman and

688 Robert K. Kanter, (Spon. by William E. Segar) Depts. of Pedlatr., Univ. Wis., Madison, WI 53792; and SUNY, Health Sc1. Ctr. at Syracuse, Syracuse, NY 13210 .

Because of its life-saving potential, teaching of emergency intravenous access including saphenous cutdown technique demands high priorfty in pediatric training programs. An inexpensive porcine foot cutdown model has been developed and tested on two pediatric housestaff populations in Syracuse, NY and Madison, WI $(\mathrm{n}=50)$.

Residents were instructed to place a $2 \mathrm{~cm}$ transverse incision 2-3 cm superior to the intersection of the first and second digits on segments of pig extremities split midline in the saggital plane. Veins may be located superficially or within deep fascial compartments. Although nonviable tissue is utilized, characteristics of the skin, connective tissue, fascia, tendons, bones, and veins provide a reasonable approximation of structures encountered in the child. Cannulation by direct insertion, by Seldinger technique, and by surgical flap and vein dilator are all serially compatible utilizing this model.

Residents exposed to this teaching device have readily completed cutdowns with minimal difficulty, with little cost, with satisfaction for the mode1, and without the need for anesthetized live animals.

Most pediatricians are likely to encounter situations where saphenous veln cutdown may be lifesaving. Initial practice utilizing the porcine foot model provides an inexpensive but realistic teaching device for this fundamental procedure.

\section{GENETICS}

A NOVEL HUMAN PHOSPHOFRUCTOKINASE GENE MAPS TO

689 CHROMOSOME 12. Patricia L. Ashley and David R. Cox. and Biochemistry/Biophysics, San Francisco.

Inherited deficiency of human phosphofructokinase,

an enzyme which catalyzes the key regulatory step in glycolysis, results in a heterogeneous group of diseases characterized by myopathy and/or hemolysis. Genetic loci coding for three types of human PFK subunit - liver $(L)$, muscle $(M)$ and platelet $(P)$ have been mapped by immunoprecipitation of protein synthesized in somatic cell hybrids to human chromosomes 21 (PFKL), 1 (PFKM) and 10 (PFKP). However, studies of the expression of individual PFK genes in human disease has been limited by lack of specific PFK DNA probes. We have used a human astrocytoma CDNA library, in conjunction with a mouse PFK CDNA probe, to isolate four human CDNA clones encoding at least three types of human PFK subunit. Complete DNA sequencing of one CDNA insert reveals $95 \%$ amino acid homology with rabbit muscle PFK (234 amino acids compared). Using a panel of mouse-human somatic cell hybrids, we have found that this human CDNA insert is derived from chromosome 12 . Mutations in this previously unrecognized human PFK gene may be responsible for the severe mental retardation observed in several recently described patients with PFK deficiency.

PROSPECTIVE DIAGNOSIS AND TREATMENT OF UREA CYCLE DISOROERS. D.Bartholomew, R. Reichel and S. Brusilow.

690 Johns Hopkins Univ School

of 31 infants known to be at risk for a urea cycle defect because of family history, 18 were treated pro spectively from birth with a disease-specific diagnostic and therapeutic protocol. Diagnosis was made on the basis of plasma citrulline and ASA levels over the first $72 \mathrm{hrs}$ of $1 \mathrm{jfe}$. Of the 7 affected infants in the prospectively treated group (OTC-3; CPS -1 AS-2; $A L-1$ ), one OTCD infant died (peak $\mathrm{NH}_{4} 850 \mathrm{uM}$ ) and 6 survived the neonatal period. (peak $\mathrm{NH}_{4} 52-197 \mathrm{UM}$ ). One QTCD survivor died at 33 mos; 5 remain on therapy (ages 18-60mos). Developmental quotients for survivors range from 55-101. 16 prospectivelytreated unaffected infants are normal at $9-60$ mos and show no $i 11$ effects from 4 days of therapy. In addition, 7 affected infants (OTC-4; CPS -2 ; AS-1) were managed outside the protocol with observation, protein-restriction and/or IV benzoate and arginine and peritoneal dialys is after the onset of symptomatic hyperammonemia. Diagnosis was made 25-90hrs after birth. All 4 OTCD infants expired (2 were untreated). The 3 survivors are now 1848 mos and remain on therapy. Peak neonatal ammonium levels ranged from 210-364uM. DQ scores in the CPSD patients are 99 and 133, while the ASD patient has a score of 55 .

We conclude: 1 ) assessment of plasma citrulline and ASA levels reliably identify affected infants within $72 \mathrm{hrs}$ of birth; 2) the prevention of neonatal hyperammonemia by prospective therapy improves survival; and 3 ) neurologic outcome is improved with early therapy in infants at risk for OTCD, CPSD, and ASD.

INCREASED SERUM HEXOSAMINIDASE A DURING PREG NANCY: IMPL ICATIONS FOR TAY - SACHS CARRIER

691 DETECTION BY SULFATED FLUOROGENIC SUBSTRATES. Thomas, HenryL, Nadler \& Michael M. Kaback* Wayne State U. Depts. of Pediatrics \& Ob/Gyn, Detroit, and *UCSD Dept of Pediatrics, San Diego.

Carrier detection of Tay-Sachs disease (TSD) in sera is unreliable during pregnancy when based on the heat inactivation method. This is due to an increase in the relatively thermostable serum hexosaminidase (Hex) P, and is overcome by testing leukocytes. Since sulfated fluorogenic substrates (MUBGICNAC-6-S \& MUBGa1NAc-6-S) are highly specilic for Hex A and provide direct determination independent of heat-labile/heat-stable ratio, we have examined their value in TSD carrier detection in pregnant women's sera. Determination of Hex activity toward sulfated substrates was carried out in isolated isozyme fractions from sera, leukocytes and fibroblasts of controls, pregnant TSD carriers and non-carriers, and TSD and Sandhoff disease patients. The results indicated that the cleavage en bloc of the N-acetylhexosamine-6-sulfate is catalyzed by an active site on the $\alpha$-subunit, since only negligible activity was associated with Hex isozymes $B, I_{1}, I_{2}$ and $P$, and $H e x S$ $(\alpha 2)$ was twice as active as $\operatorname{Hex} A\left(\alpha \beta_{a} \beta_{b}\right)$. The results also indicated that in addition to serum Hex $p$ there is an increase in serum Hex $A$ which starts during the second trimester of pregnancy. We conclude that accurate TSD carrier detection in serum during pregnancy may be possible if gestational age-matched controls are used. 